VOL. $13(1975), 317-318$.

\title{
Balanced subgroups of abelian groups
}

\section{Roger H. Hunter}

In 1967, Hill extended Ulm's classification theorem for reduced countable $p$-groups to the class of totally projective abelian p-groups introduced by Nunke. Hill has also shown that the class of totally projective abelian $p$-groups is the class of projectives relative to a class of short exact sequences (called balanced by Fuchs) of p-groups.

In this thesis, balanced sequences of arbitrary abelian groups are defined and the corresponding classes of projectives and injectives studied. The properties of balanced sequences are explored and it is shown that the new definition specialises, in the case of torsion groups, to that of Fuchs. The class of injectives relative to balanced sequences is shown to coincide with the well known class of pure injectives.

In order to study the balanced projectives, a theory of mixed abelian groups of torsion free rank $I$ is developed, considering them as extensions of a cyclic group by a torsion group. It is shown that a balanced projective is a direct summand of a direct sum of groups having torsion free rank at most 1 , where each group of torsion free rank at most 1 involved is an extension of a cyclic group by a totally projective torsion group.

Certain closure properties enjoyed by the class of totally projective p-groups are shown to fail for the class of balanced projectives. However, it is shown that the class of projectives relative to a subclass of the class of balanced sequences has the required closure properties and that this new class is only slightly larger than the class of balanced projectives.

The thesis concludes with an investigation of a number of questions of

Received 25 February 1975. Thesis submitted to the Australian National University, March 1975. Degree approved, June 1975. Supervisors: Dr K.M. Rangaswamy, Dr L.G. Kovács. 
the following type. Given a class $X$ of abelian groups ( of all torsion groups), for what abelian groups $Y$ is it true that all balanced extensions of groups in $X$ by $Y$ (or of $Y$ by groups in $X$ ) must split? 